\title{
INTERFERENCE OF 802.11B WLAN AND Bluetooth: ANAlysis AND PERformance EVALUATION
}

\author{
Anil Mathew, Nithin Chandrababu, Khaled Elleithy, Syed Rizvi \\ Department of Computer Science and Engineering, University of Bridgeport, \\ Bridgeport, CT 06604 \\ \{amathew, nchandra, elleithy, srizvi\}@bridgeport.edu
}

\begin{abstract}
IEEE 802.11 and Bluetooth, these two operating in the unlicensed $2.4 \mathrm{GHz}$ frequency band are becoming more and more popular in the mobile computing world. The number of devices equipped with IEEE 802.11 and Bluetooth is growing drastically. Result is the number of co-located devices, say within 10 meters, grown to a limit, so that it may causes interference issues in the $2.4 \mathrm{GHz}$ radio frequency spectrum. Bluetooth supports both voice synchronous connection oriented (SCO) data and asynchronous connection less (ACL) packets. In this paper, we investigate the interference issues of $2.4 \mathrm{GHz}$ frequency band. In addition, this paper presents a new Bluetooth voice packet Synchronous Connection Oriented with Repeated Transmission (SCORT) scheme to optimize the performance of $2.4 \mathrm{GHz}$ frequency band by minimizing the interference between Bluetooth and 802.11 wireless networks. For the sake of experimental verifications, we provide a comprehensive simulation results using Matlab Simulink.
\end{abstract}

\section{KEYWORDS}

Bluetooth, 802.11 Wireless Networks, Mobile Nodes, Interference.

\section{INTRODUCTION}

The growth of wireless networks has transformed our daily life into such a situation that we cannot think of a life without devices like computers, mobile phones etc. The wireless networks that interconnect these devices are adding more and more nodes into it each minute [2]. These devices communicate with each other using many popular standards developed by IEEE and such other groups [1].

The most popular among these communication standards are IEEE 802.11 or Wi-Fi and the Bluetooth. Almost $75 \%$ of the devices in the mobile computing world are equipped with either one of these or both. These technologies use the radio frequency for communication [3,4]. The Bluetooth operates in $2.4 \mathrm{GHz}$ ISM band. Unfortunately, IEEE 802.11 also operates in the same $2.4 \mathrm{GHz}$ ISM band that causes significant interference. There are different versions of IEEE 802.11 like $802.11 \mathrm{a}, 802.11 \mathrm{~b}, 802.11 \mathrm{~g}$, and $802.11 \mathrm{n}$ to name a few. In this paper we consider $802.11 \mathrm{~b}$ which operates in the $2.4 \mathrm{GHz}$ ISM band as shown in Fig. 1 . When a node using IEEE $802.11 \mathrm{~b}$ as a wireless standard wants to send a packet through the network, it uses the carrier sense protocol running at the medium access control (MAC) layer to determine whether the medium is occupied or idle [5].

If it finds the medium idle (i.e., none of the other stations sensing any RF energy in the channel), it issues Clear to Send (CTS) request packet [6] to the destination node. If destination node wants to communicate, it sends back a small Ready to Send (RTS) packet to the sending node. When a sending node receives a positive acknowledgement from the destination node (i.e., RTS packet), both sending and receiving nodes can start communicating with each other by exchanging the regular data packets. Using the same technique, while another co-located IEEE 802.11b network tries to send the packet, it will postpone the transmission [14]. 


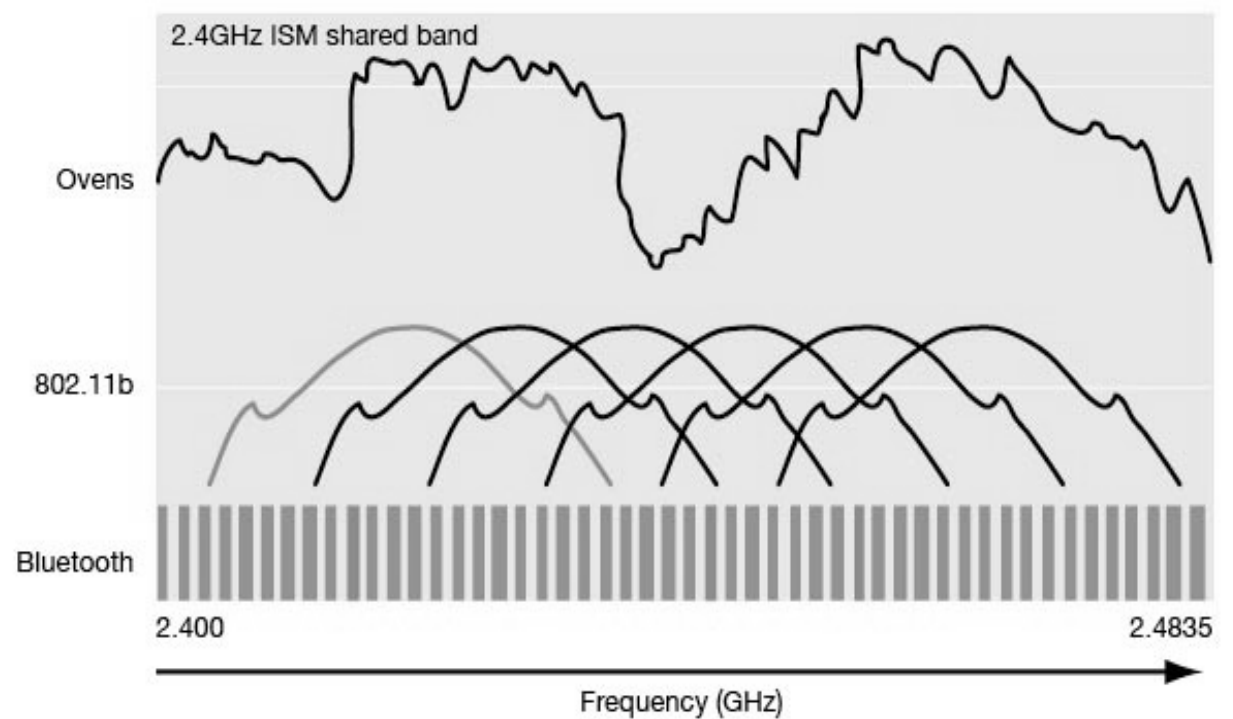

Figure 1: $2.4 \mathrm{GHz}$ ISM Spectrum

This technique provides a good resolution for mutual interference between co-located IEEE 802.11 networks. However, when it comes to a co-located Bluetooth and IEEE 802.11 network, they just do not communicate with each other. There is a definite chance of collision when they use the same channel at a particular time.

A Bluetooth device may haphazardly begin transmitting packets while an IEEE 802.11 device is sending a frame $[8,9]$. This may results in interference which forces the IEEE 802.11 station to retransmit the frame when it realizes that the destination station is not going to send back an acknowledgment. This lack of coordination is the basis for interference between Bluetooth and $802.11[10,15]$.

The objective of this paper is to build a simulation model and study the impact of interference between IEEE $802.11 \mathrm{~b}$ and Bluetooth. We also investigate about a new Bluetooth voice packet to reduce interference which is proposed by IEEE working group on co-existence.

This paper is organized as follows: Section 2 presents related work. Section 3 presents the simulation model with a brief discussion. In Section 4, we present "SCORT" the new voice packet. Testing of the model and results are presented in Section 5. Finally we conclude in Section 6.

\section{RELATED WORK}

Vilovic et al. [16] analyzed the wireless network performance with 802.11 devices and Bluetooth devices co-existing. Jayaparvathy et al. [17] did a delay performance analysis of 802.11 networks. Garetto et al. analyze the performance of 802.11 WLANs that employ the Distributed Coordination Function (DCF). They consider contending stations within radio proximity and investigate the case in which stations operate under non-saturated conditions [18].

Bluetooth device can send both voice and data packets through a radio channel with a data rate of $1 \mathrm{Mbps}$. Bluetooth are a short range personal area network (PAN) [12]. Its operating range is normally no more than 10 meters. Transmitting power of a Bluetooth Tx is very low. It is just 1 $\mathrm{mW}$. Bluetooth uses Gaussian Frequency Shift Keying (GFSK) modulation technique [11]. Bluetooth also uses Frequency Hopping Spread Spectrum (FHSS) technique to reduce 
interference from other devices operating in the same frequency spectrum. Interference in Bluetooth system can be recovered or sometimes avoided using various coexistence techniques [12, 13, 14, and 15]. Figure 2 represents the utilization of time slot in Bluetooth. In this paper, we consider synchronous connection oriented with Repeated Transmission (SCORT) to reduce the effect of interference in Bluetooth synchronous connection oriented (SCO) voice links.

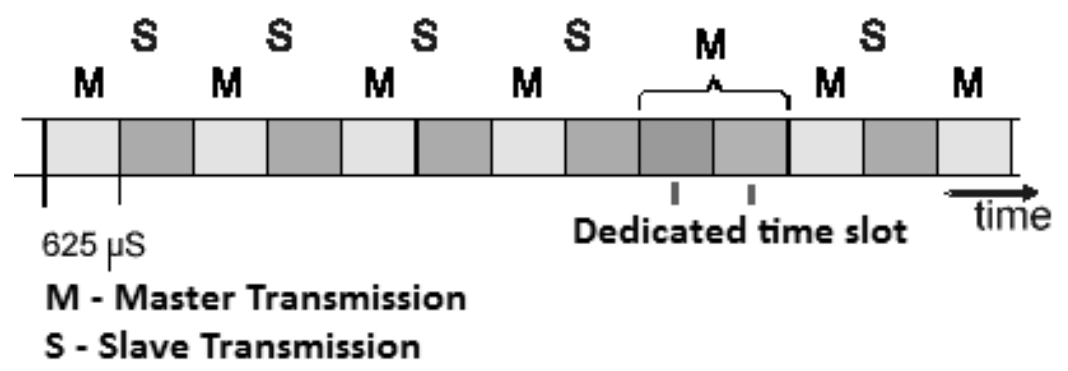

Figure 2. Bluetooth time Slot

A time division multiplexing technique divides the channel into slices of $625 \mu$ s slots as shown in Figure 5. A new hop frequency is used for each slot. Bluetooth supports both voice and data transmission. Bluetooth voice transmission is called SCO where as data transmission is refereed as asynchronous connection less (ACL). Bluetooth SCO link is established between a master device and a slave device in the Piconet as shown in Figure 3. SCO link uses reserved slots to communicate. Bluetooth master device use these reserved slots to maintain the communication. Bluetooth establishes an ACL link to transmit data. Unlike SCO, ACL links can be established between one master device and up to seven slave devices. ACL packets are transmitted in the free slots after SCO transmission. An ACL packet can be occupied up to one, three or five slots. All ACL packets other than Broadcast from master are acknowledged.

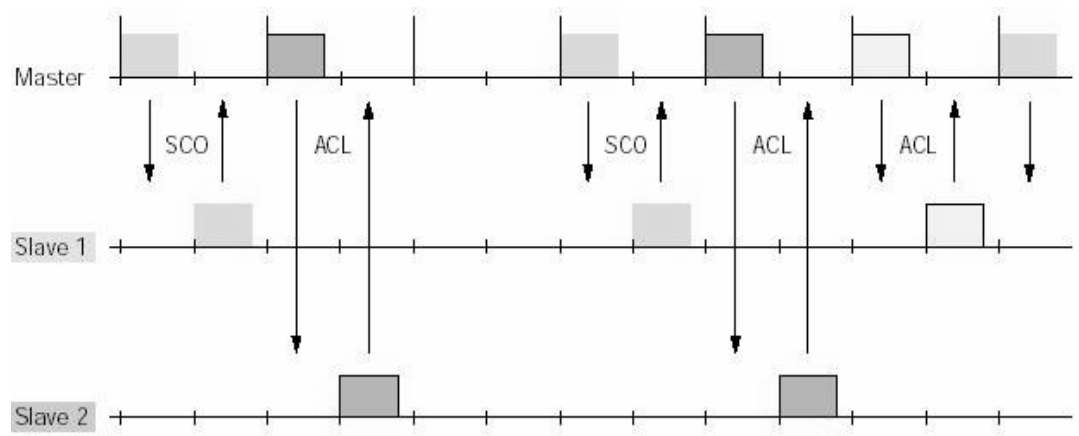

Figure 3. Bluetooth SCO \& ACL

\subsection{Synchronous Connection Oriented (SCO) Link}

Bluetooth voice transmission is done by SCO. The SCO link is a symmetric point to point voice link for sending and receiving voice packets at regular intervals of time. The 
SCO packets are transmitted in only every sixth slot. This period of time is equal to 3.75 $\mathrm{ms}$. The return path of transmission from the slave to master takes place on the next slot. Bluetooth can support a maximum of up to three voice calls at the same time. In Figure 4, T1, T2, and $T 3$ are the transmit slots for each SCO master link. Slots $R 1, R 2$, and $R 3$ are the return path for the slaves.

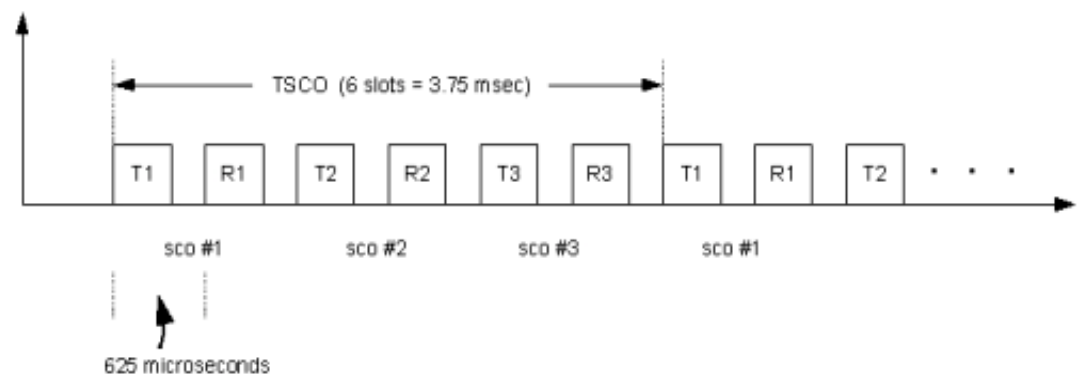

Figure 4. Bluetooth SCO voice slot

A master device initializes and controls the SCO link. Up to a maximum of three SCO links can be maintained by a master device at the same time. When a master device sends SCO a packet in a slot, the slave device sends back in the following slot. This shows that the transmission of packet is symmetric (i.e., data rate is same in both directions). The length of the Bluetooth-SCO packet is always one slot. There is no acknowledgement for SCO packets. SCO packet transmission happens always in reserved slots at regular time intervals, every two, four or six slots.

There are different types of SCO voice packets like HV1, HV2, and HV3. HV1 carries 10 data bytes and is transmitted every 2 slots, HV2 carries 20 data bytes and is transmitted every 4 slots and HV 3 carries 30 data bytes and is transmitted every 6 slots.The data rate of HV1, HV2, HV3 packets are 64Kbps. HV1 and HV2 uses $1 / 3$ and $2 / 3$ rate forward error correcting (FEC) mechanisms, respectively. There is no FEC in HV3.

\subsection{Asynchronous Connection Less (ACL) Link}

Bluetooth data transmission is called ACL which is different from SCO transmission in many respects. In data transmission there is no margin for error allowed. If an error occurs, those packets must be transmitted again. Different techniques can be used to implement it. In the case of Bluetooth ACL transmission, the system will wait for acknowledgement from the receiver. It will send the packets repeatedly till an acknowledgement is received. The receiver will check the packet and verify the cyclic redundancy code (CRC) to make sure that the packet is received correctly. In ACL-Tx the throughput (in bps) must be checked. The bit error rate (BER) does not matter much. The throughput may go down if there is a significant amount of retransmission is required. The receiver sets the Automatic Repeat Request Number (ARQN) bit in the header part of the packet. It will then send it to transmitter in the return path packet. That is how receiver sends an ACK. By checking the ARQN, transmitter senses if the transmission was successful. If the value of ARQN is 1 , it means a successful transmission, and if ARQN is 0 it means a failed transmission. In the case of a one way communication (master-to-slave), the slave sends back a dummy packet in the next slot. NULL packet or dummy packet does not have any payload. Figure 5 shows the DM1 packet being transmitted in the first slot, and the slave replying with a NULL packet containing the ACK in the immediately following slot. The master then transmits again in the next slot. 


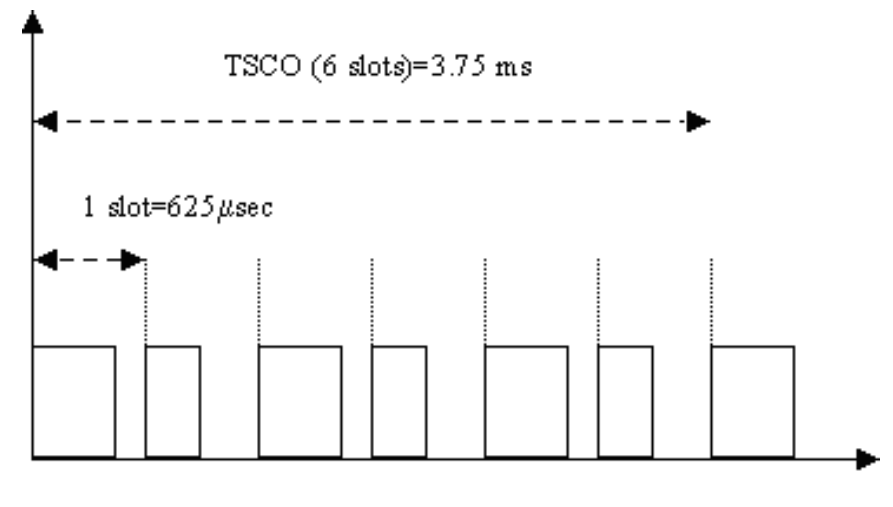

DMl NULL DMl HULL DMl HULL DMl

Figure 5. Asynchronous Connection Less (ACL) link

\section{Bluetooth Simulation Model}

Figure 6 shows the simulation model of the network in MATLAB Simulink. This model simulates Bluetooth in full duplex communication mode. We use two similar devices each with a transmitter and receiver. One of them should be set as master and the other as the slave. Other than two Bluetooth devices, we also have an $802.11 \mathrm{~b}$ packet generating block as an interference source, error reading meters and instrumentation.

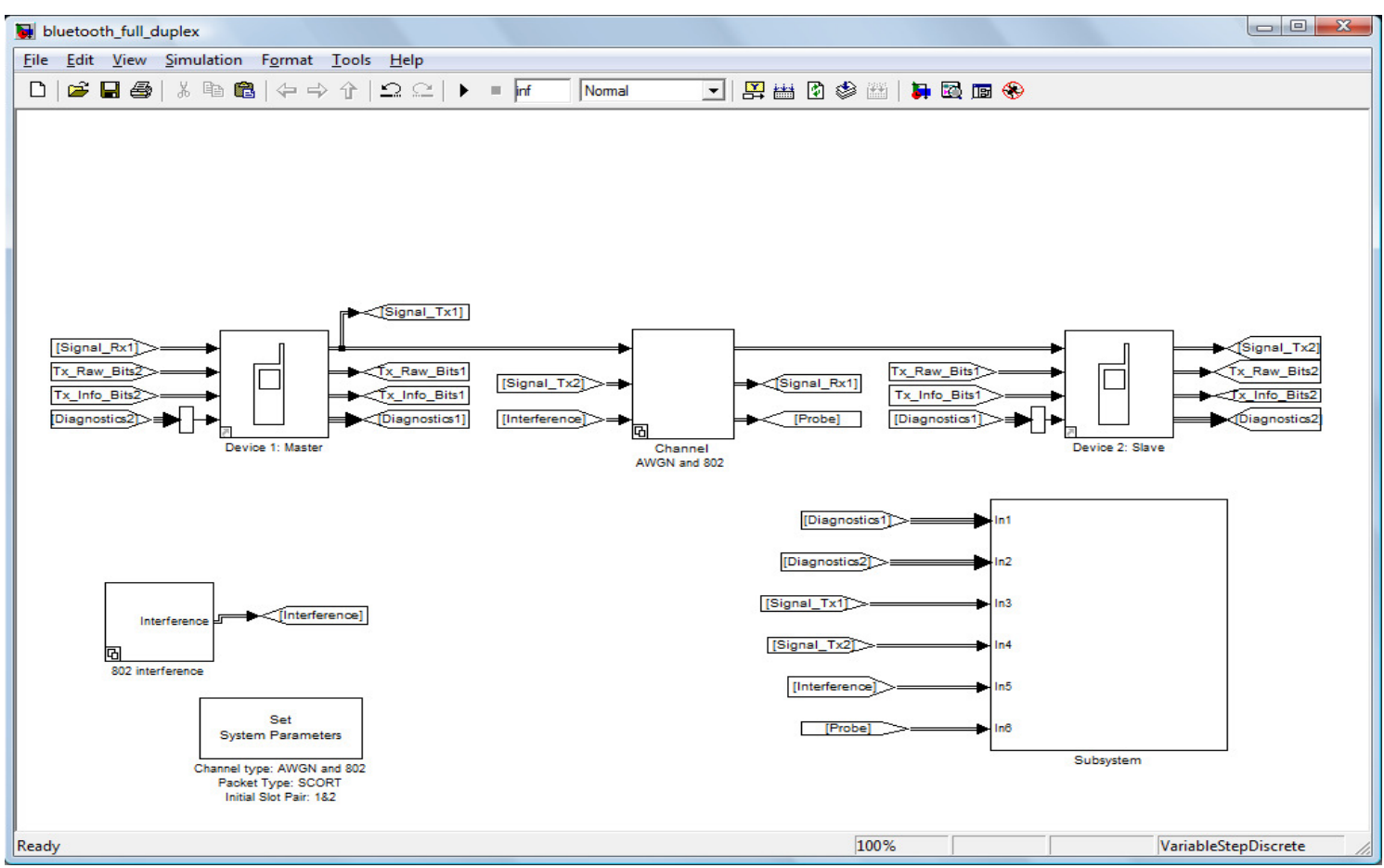

Figure 6. Bluetooth Interference Simulation Model 


\subsection{Transmitter Design}

The transmitter mode is shown in Figure 7. Figure7 shows the state flow diagram of the data transmission. The transmitted model processes both data and voice input. It also performs Header Error Control (HEC) using FEC. Buffering and modulation is also done at the transmission side as shown in Figure 7. Frequency hopping is the transmission modulation technique. When the "ACL_packets" is entered, the transition to "Transmit_blank_packet" will happen. The "Enable_Audio=0" \& "Get_blank_Packet=1" actions activates to disable audio and to generate a new data packet. When the next slot is about to transmit, the transmitter checks the status of ARQN bit returned from the receiving device.

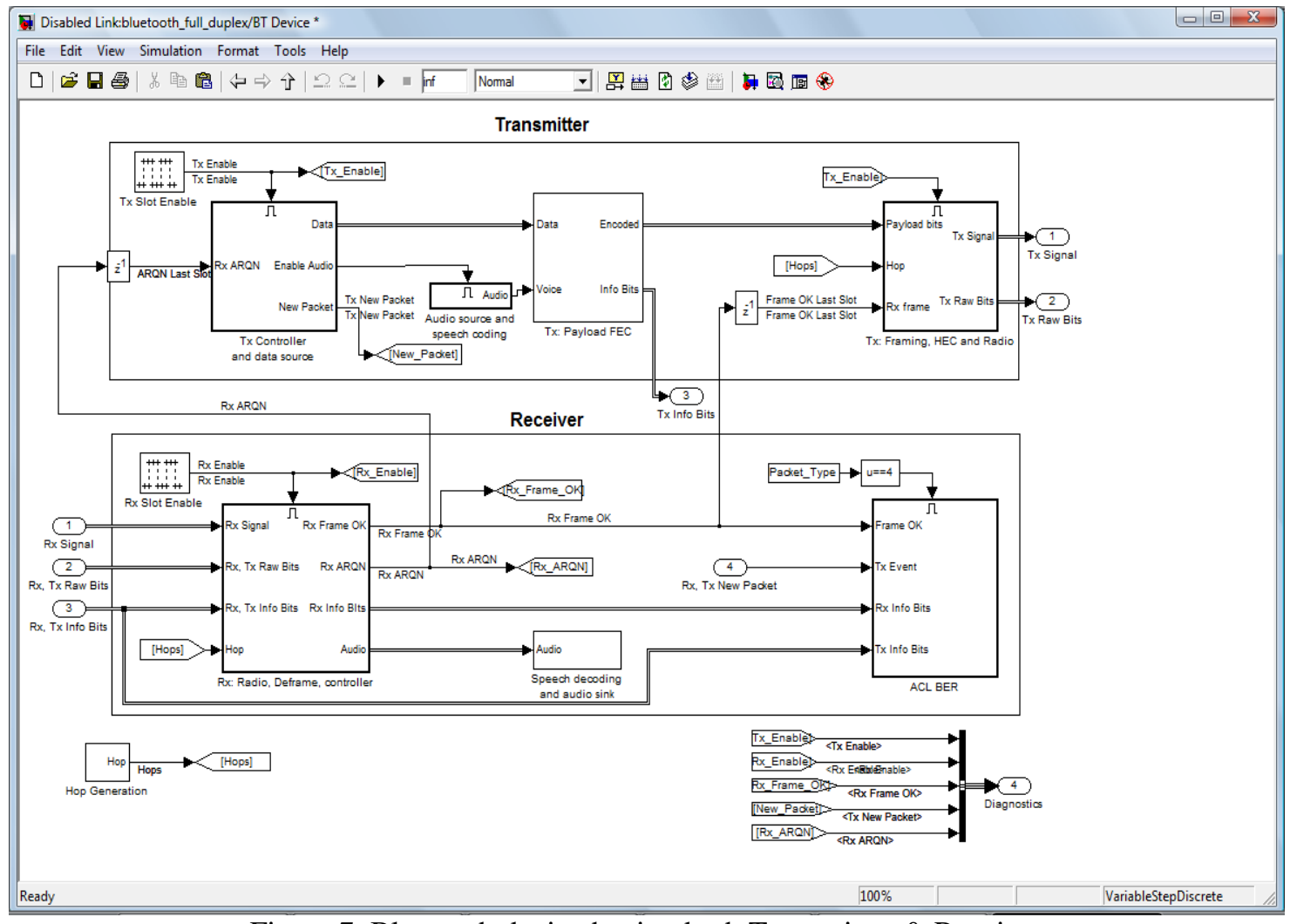

Figure 7. Bluetooth device having both Transmitter \& Receiver

If it is in "Transmit_blank_Packet" ARQN is one, it stays in the state and transmits another new packet. If ARQN is zero, it shifts to the "Re_Transmit_Packet". This simulation model uses frame based processing. It can transmit samples having high number of frames in each step of the simulation. This technique enables quick simulation of digital systems. In this particular model, a top sample rate of $100 \mathrm{MHz}$ is used.

Figure 8 shows the state flow diagram of the data transmission. When the "ACL_packets" is entered the transition to "Transmit_blank_packet" will happen. The "Enable_Audio $=0$ " \& "Get_blank_Packet $=1$ " actions activates to disable audio and to generate a new data packet. When the next slot is about to transmit, the transmitter will check the status of ARQN bit returned from the receiving device. If it is in "Transmit_blank_Packet" ARQN is one, it stays in the state and transmits another new packet. If ARQN is zero, it shifts to the "Re_Transmit_Packet". If the transmitter is in "Re_Transmit_Packet", and ARQN is one, it shifts to "Transmit_blank_Packet" else it will not shift and will stay in "Re_Transmit_Packet". 


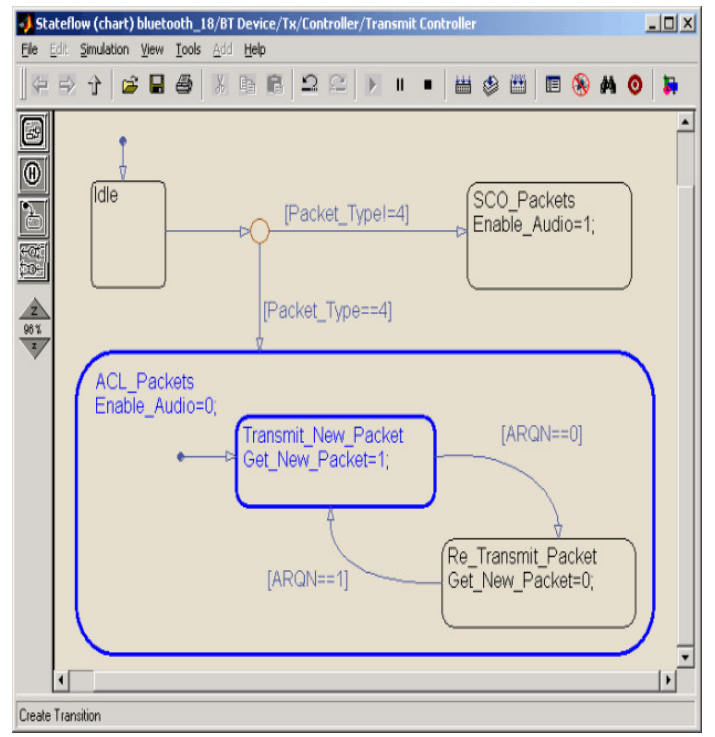

Figure 8. Transmitter state flow diagram

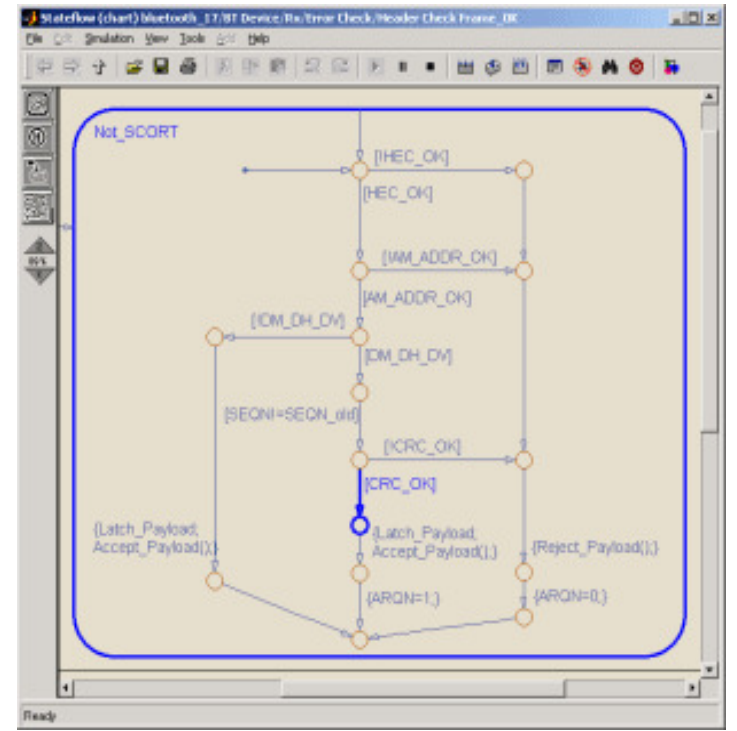

Figure 9. Receiver state flow diagram

\subsection{Receiver Design}

The state flow diagram of receiver is shown in Figure 9. It can be seen in Figure 9 that the receiver waits a new packet all the time. When it senses the arrival of a packet it will register the arrival. It will also make sure the decoder is enabled. The above mentioned sequence of events is triggered because of the detection of an arriving packet. The receiver has to make a number of decisions to make sure whether the received packet is correct or incorrect.

A DM1 packet will be checked for integrity. The receiver performs a HEC. The address is also verified. The receiver makes sure the packet is new and is not a duplicate. It also checks the CRC.

If all these checks are correct, the packet will be accepted else the packet will be rejected. This happens in the case of a repeated packet arriving or in the case of its CRC failing. This flow diagram is implemented in State flow semantics as shown in Figure 8. This image captured during a simulation, illustrates the animation provided with State flow which highlights the decision path (in bold) through the flow chart.

\subsection{Channel and Interferer Modeling}

The $802.11 \mathrm{~b}$ channel bandwidth is approximately $22 \mathrm{MHz}$. The Simulation model has a block which produces signals in this bandwidth. This block can be configured to specify mean packet rate, packet length, power, and frequency location in the ISM band. This block is then connected to the channel where the distance between the interference source and Bluetooth system can be varied. Figure 10 shows the addition of $802.11 \mathrm{~b}$ interference into the channel. We use this model in our experimental verifications to determine the behavior of added interference.

\section{Coexistence Solution - SCORT Voice Transmission}

The coexistence task group working on coexistence has suggested the use of a special voice packet to fight interference. The synchronous connection-oriented with Repeated Transmission (SCORT) packet achieves more robust transmission by replacing bit-level redundancy with packet-level redundancy. The state flow diagram of SCORT is presented in Figure 11. It works 


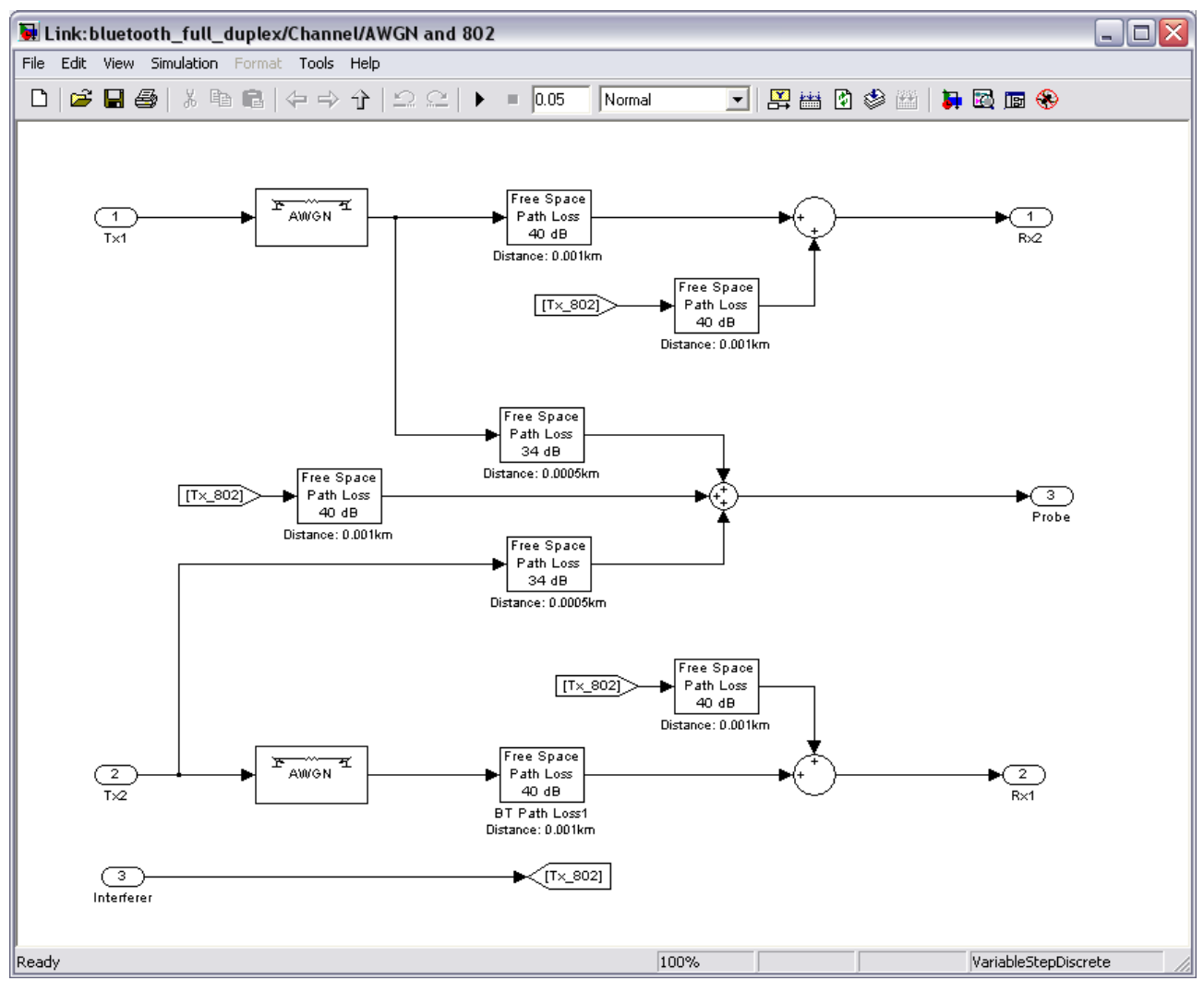

Figure 10. 802.11b Interference Source added

by repeating the transmission of the same packet three times in one SCO interval. SCORT does not have any error correction. SCORT is transmitted every second time slot. As the same packet is being transmitted three times in a row, only one voice link will be there which is a full duplex link. If interference destroys the transmission during first slot, there are still three other slots or opportunities to communicate the packet. This provides an improvement for frame-error rate (FER) in an interference scenario. It does not affect the BER of the payload.

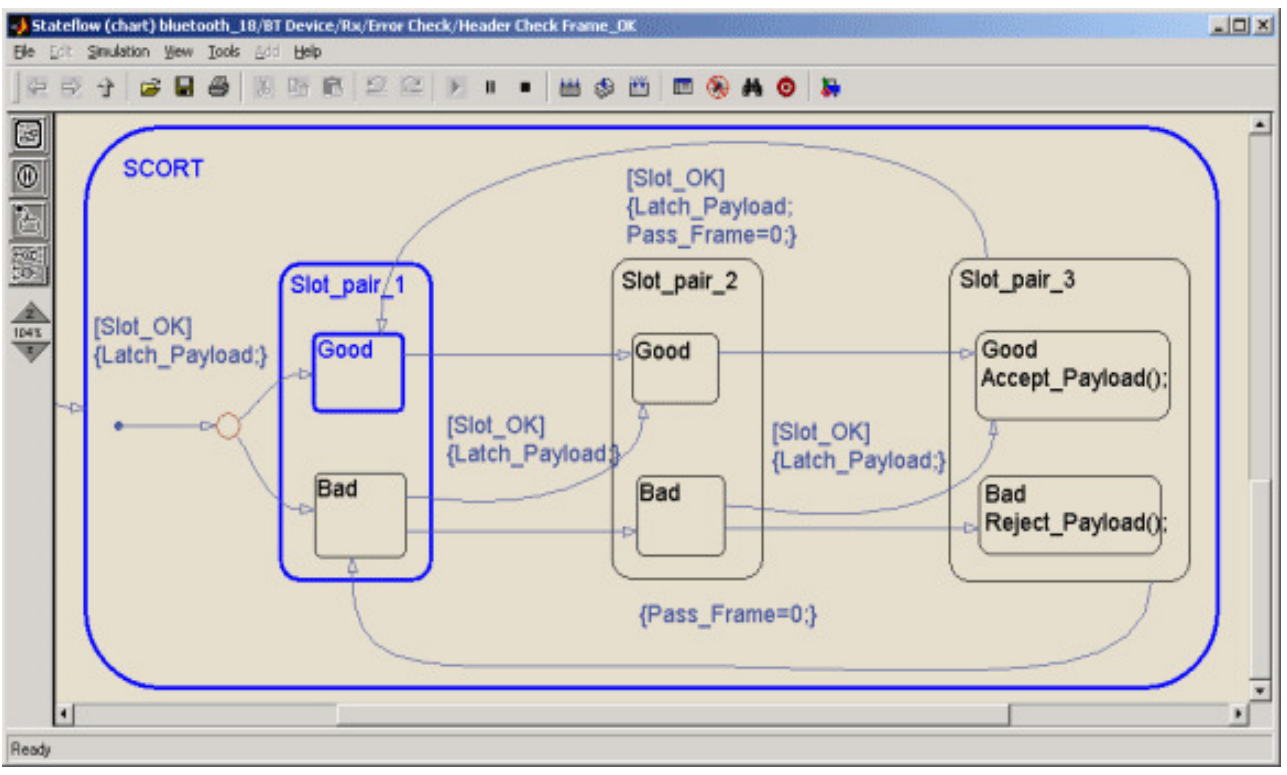

Figure 11. SCORT State Flow Diagram 


\section{EXPERIMENTS AND RESULTS}

Using the above models, we perform a series of tests to evaluate the performance of a Bluetooth system under interference. We used DM1 packet type to check the performance of ACL transmission. Packet types HV1, HV2 and HV3 are used to evaluate SCO performance. Finally we used SCORT packet type to compare its performance with HV1, HV2 and HV3.

Figure 12 shows the Bluetooth system throughput. The throughput (in kbps) has been evaluated by varying the distance between the device and the interference source. It should be noted in Figure 12 that a consistent values of throughput is achieved with respect to a constant increase in the distance between the Bluetooth devices. From Figure 12, we can see that the throughput of a Bluetooth system is about $128 \mathrm{kbps}$ without $802.11 \mathrm{~b}$ interference source.

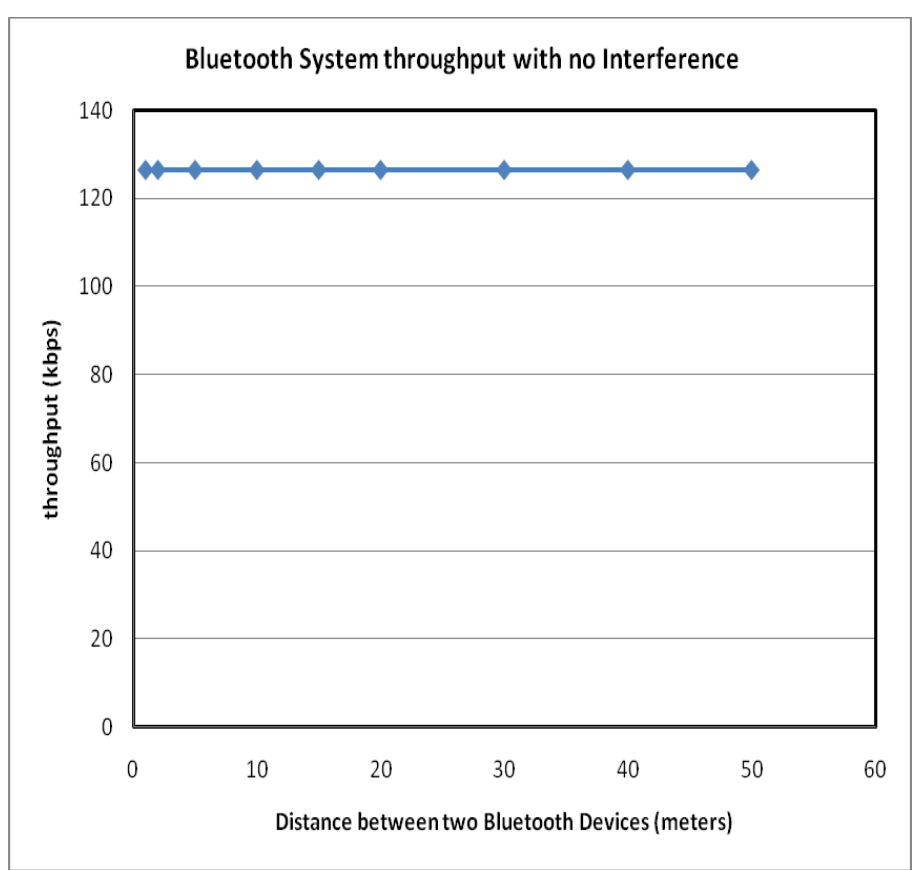

Figure 12. Bluetooth system throughput with respect to the distance between Bluetooth devices

Figure 13 shows the reduction in the throughput when $802.11 \mathrm{~b}$ interfering source come closer to the Bluetooth system. Figure 14 demonstrates the BER performance with respect to Eb/No. It should be noted in Figure 14 that the BER decreases linearly over the values of Eb/No. However, the BER divergence in Figure 14 is very rapid and acceptable for a maximum value of $\mathrm{Eb} / \mathrm{No}$.

For Figure 15, we measured the difference in FER when using a SCORT voice packet rather than the regular HV1, HV2, and HV3 packets. From Figure 15, we can see that when using SCORT packets, there is a considerable reduction in the FER.

\section{Conclusions}

This paper presented a model for the interference of both IEEE $802.11 \mathrm{~b}$ and Bluetooth. Our analysis shows that interference increases significantly with respect to an increase in the number of participating devices. Techniques such as SCORT are a big leap in the future for such networks. Our simulation results suggest that the use of SCORT packets can minimize the effect 


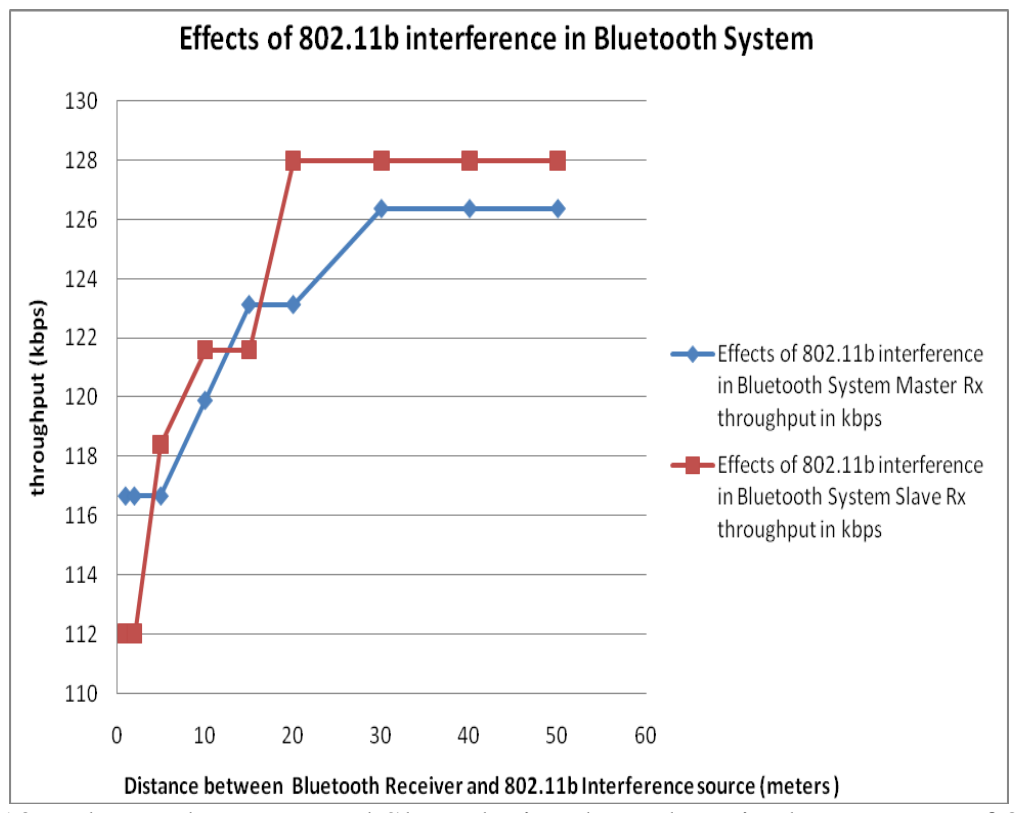

Figure 13. Bluetooth Master and Slave device throughput in the presence of $802.11 \mathrm{~b}$

of interference between 802.11 wireless network and Bluetooth devices. In the future, it is expected that the wireless industry will mature in such a way that smooth data and voice transmission will be achieved with a minimal interference.

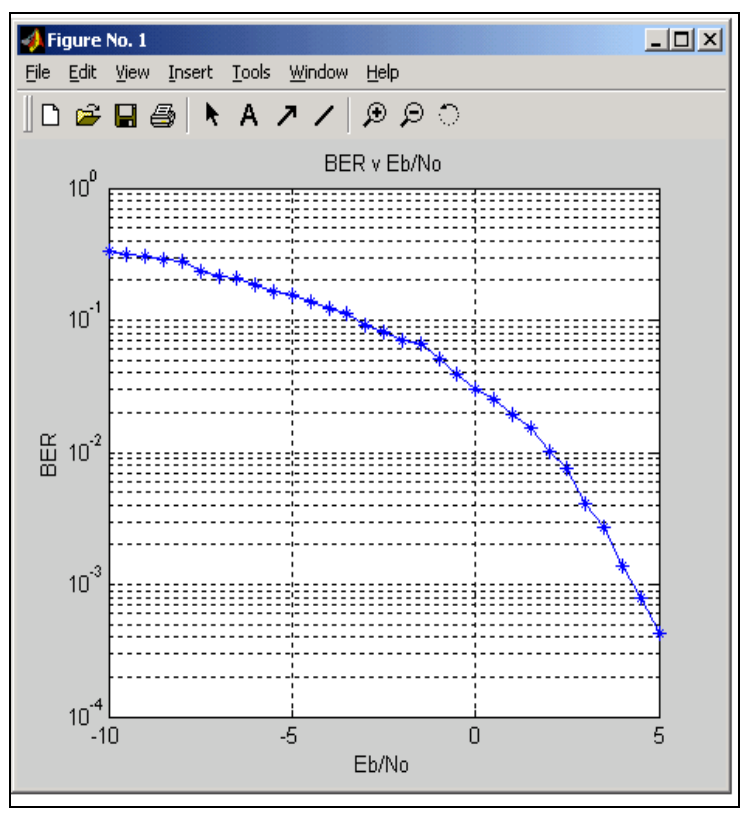

Figure 14: BER versus Eb/No

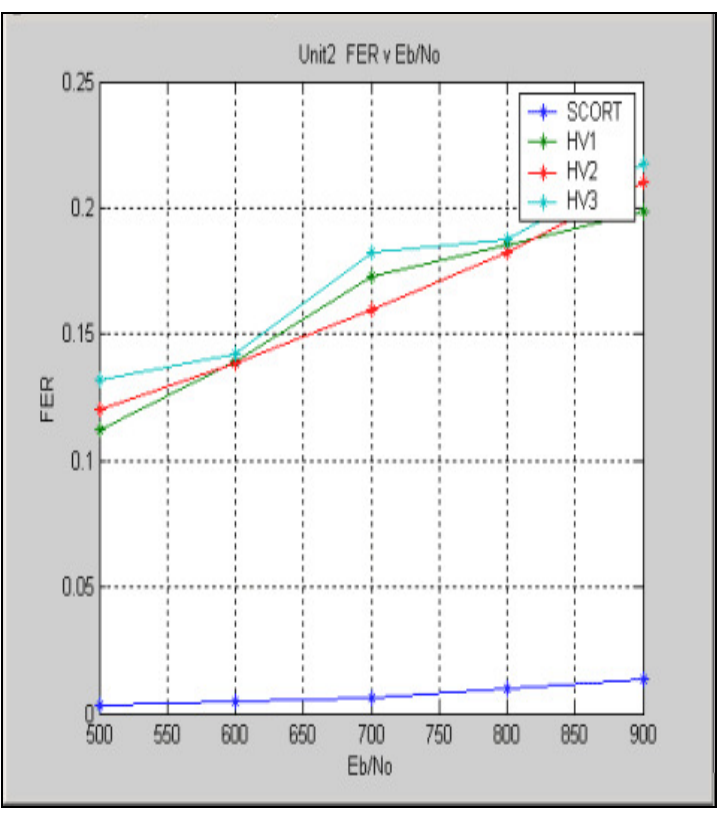

Figure 15: BER versus large values of Eb/No 


\section{REFERENCES}

[1] Golmie, N. (2004). Bluetooth dynamic scheduling and interference mitigation, Mobile Networks and Applications, Vol. 9, Issue 1, pp. 21-31.

[2] Golmie, N.; Dyck, R. E.; Soltanian, A. (2001). Interference of Bluetooth and IEEE 802.11: simulation modeling and performance evaluation, Proceedings of the 4th ACM International Workshop on Modeling, Analysis and Simulation of Wireless and Mobile Systems, pp. 11- 18.

[3] Golmie, N.; Dyck, R. E.; Soltanian, A. ; Tonnerre, A. ; Rébala, O. (2003). Interference evaluation of Bluetooth and IEEE 802.11b systems, Journal of Wireless Networks, Vol. 9, Issue 3, pp. 201 211.

[4] Song, M.; Shetty, S.; Gopalpet, D. (2007). Coexistence of IEEE 802.11b and Bluetooth: an integrated performance analysis, Mobile Networks and Applications, Vol. 12, Issue 5, pp. 450 459.

[5] Wadhwa, M.; Song, M. (2006). Performance of IEEE 802.11b devices in the presence of adaptive frequency hopping enabled Bluetooth devices, Proceedings of the 4th IEEE International Conference on Information Technology: Research and Education, pp. 74 - 48.

[6] Subramanian, V.; Ramakrishnan, K.; Kalyanaraman, S.; Lusheng, J. (2006). Impact of interference and capture effects in 802.11 wireless networks on TCP, Proceedings of the 2nd International Workshop on Wireless Traffic Measurements and Modeling.

[7] Mathew, A.; Chandrababu, N.; Elleithy, K.; Rizvi, S. (2009). IEEE 802.11 \& Bluetooth interference: simulation and coexistence, 7th Annual Conference on Communication Networks and Services Research, pp. 217 - 223.

[8] Matthew, B. (2009). Wi-Fi (IEEE 802.11b) and Bluetooth coexistence issues and solutions for the 2.4 GHz ISM band, Texas Instruments.

[9] Cordeiro, C.; Abhyankar, S.; Toshiwal, R.; Agarwal, R. (2003). A novel architecture and coexistence method to provide global access to/from Bluetooth WPANs by IEEE 802.11 WLANs, Proceedings of IEEE International Perform Computer Communication, pp 23-30.

[10] Chek, M.; Kwok, Y. (2007). Design and evaluation of practical coexistence management schemes for Bluetooth and IEEE 802.11b systems, International Journal of Computer and Telecommunications Networking, Vol.51, No.8, pp. 2086 - 2103.

[11] Santivanez, C.; Ramanathan, R.; Partridge, C.; Krishnan, R.; Condell, M.; Polit, S. (2006). Opportunistic spectrum access: challenges, architecture, protocols, Proceedings of the 2nd Annual International Workshop on Wireless Internet, Vol. 220, Article No. 13.

[12] Kamerman, A. (2000). Coexistence between Bluetooth and IEEE 802.11 CCK: solutions to avoid mutual interference, IEEE P802.11 Working Group Contribution, IEEE P802.11-00/162r0.

[13] Pasolini, G. (2004). Analytical investigation on the coexistence of Bluetooth Piconets, IEEE Communications Letters, Vol. 8, No. 3, pp. 144-146.

[14] Shellhammer, S. (2001). Collaborative coexistence mechanism: TDMA of Bluetooth and 802.11, IEEE 802.15-01/025r0.

[15] Lansford, J.; Nevo, R. (2000). MEHTA: A method for coexistence between co-located 802.11b and Bluetooth systems, IEEE 802.15-00/036r0.

[16] Vilovic, I.; Zovko-Cihlar, B. (2004). Performance analysis of wireless network using Bluetooth and IEEE 802.11 devices, In Proceedings of the 46th International Symposium in Electronics in Marine, pp. 235 - 240.

[17] Jayaparvathy, R.; Rajesh, S.; Anand, S.; Srikanth, S. (2003). Delay performance analysis of 802.11 communications, In Proceedings of 9th Asia-Pacific Conference on Communications, pp. 223-226.

[18] Garetto, M.; Chiasserini, C.-F. (2005). Performance analysis of 802.11 WLANs under sporadic traffic, Lecture Notes in Computer Science, pp.1343-1347. 